\title{
Distributed Massive MIMO: Random Access, Extreme Multiplexing and Synchronization
}

Unnikrishnan Kunnath Ganesan 

Linköping Studies in Science and Technology

Licentiate Thesis, No. 1923

\title{
Distributed Massive MIMO: \\ Random Access, Extreme \\ Multiplexing and Synchronization
}

\author{
Unnikrishnan Kunnath Ganesan
}

\section{SOMMUNICATION Ŝ̄STEMS}

Division of Communication Systems

Department of Electrical Engineering (ISY)

Linköping University, 58183 Linköping, Sweden

www.commsys.isy.liu.se

Linköping 2022 
This is a Swedish Licentiate Thesis.

The Licentiate degree comprises 120 ECTS credits of postgraduate studies.

(oc) EY-NG This work is licensed under a Creative Commons AttributionNonCommercial 4.0 International License.

https://creativecommons.org/licenses/by-nc/4.0/

Distributed Massive MIMO: Random Access, Extreme Multiplexing and Synchronization

(C) 2022 Unnikrishnan Kunnath Ganesan, unless otherwise stated.

ISBN 978-91-7929-220-1(print)

ISBN 978-91-7929-221-8(PDF)

ISSN 0280-7971

URL https://doi.org/10.3384/9789179292218

Printed in Sweden by LiU-Tryck, Linköping 2022 


\section{Abstract}

The data traffic in wireless networks has grown tremendously over the past few decades and is ever-increasing. Moreover, there is an enormous demand for speed as well. Future wireless networks need to support three generic heterogeneous services: enhanced mobile broadband(eMBB), ultrareliable low latency communication (URLLC) and massive machine type communication (mMTC). Massive MIMO has shown to be a promising technology to meet the demands and is now an integral part of $5 \mathrm{G}$ networks.

To get high data rates, ultra densification of the network by deploying more base stations in the same geographical area is considered. This led to an increase in inter-cell interference which limits the capacity of the network. To mitigate the inter-cell interference, distributed MIMO is advocated. Cell-free massive MIMO is a promising technology to improve the capacity of the network. It leverages all the benefits from ultra densification, massive MIMO, and distributed MIMO technologies and operates without cell boundaries.

In this thesis, we study random access, extreme multiplexing capabilities, and synchronization aspects of distributed massive MIMO. In Paper A studies the activity detection in grant-free random access for mMTC in cell-free massive MIMO network. An algorithm is proposed for activity detection based on maximum likelihood detection and the results show that the macrodiversity gain provided by the cell-free architecture improves the activity detection performance compared to co-located architecture when the coverage area is large.

RadioWeaves technology is a new wireless infrastructure devised for indoor applications leveraging the benefits of massive MIMO and cell-free massive MIMO. In Paper B, we study the extreme multiplexing capabilities of RadioWeaves which can provide high data rates with very low power. We observe that the RadioWeaves deployment can spatially separate users much better than a conventional co-located deployment, which outweighs the losses caused by grating lobes and thus saves a lot on transmit power.

Paper C studies the synchronization aspect of distributed massive MIMO. 
We propose a novel, over-the-air synchronization protocol, which we call as BeamSync, to synchronize all the different multi-antenna transmit panels. We also show that beamforming the synchronization signal in the dominant direction of the channel between the panels is optimal and the synchronization performance is significantly better than traditional beamforming techniques. 


\section{Populärvetenskaplig Sammanfattning}

Efterfrågan på data ökar ständigt och kravet på hastighet har ökat enormt. Framtida trådlösa nätverk behöver stödja tre generiska heterogena tjänster: enhanced mobile broadband (eMBB), ultra-reliable low latency communication (URLLC) och massive machine type communication (mMTC). Massiv MIMO har visat sig vara en lovande teknik för att möta efterfrågan och är nu en integrerad del av 5G-nätverket.

För att få höga datahastigheter övervägs extrem förtätning av nätverket genom att distribuera fler basstationer i samma geografiska område. Detta leder till en ökning av intercellinterferens men systemets kapacitet begränsas av intercellinterferensen. För att mildra intercellinterferensen förespråkas distribuerad MIMO. Cellfri massiv MIMO utnyttjar alla fördelar från ultraförtätning, massiv MIMO och distribuerad MIMO-teknik och fungerar utan cellgränser.

I denna avhandling studerar vi random access, extrema multiplexerings möjligheter och synkroniseringsaspekter av distribuerad massiv MIMO. I Paper A studeras aktivitetsdetekteringen i grant-free random access för mMTC i cellfria massiv MIMO-nätverk. En algoritm föreslås för aktivitetsdetektering baserad på maximum likelihood-metoden och resultaten visar att den makrodiversitetsvinst som tillhandahålls av den cellfria arkitekturen förbättrar aktivitetsdetekteringsprestandan jämfört med samlokaliserad arkitektur när täckningsområdet är stort.

RadioWeaves-teknologi är en ny trådlös infrastruktur utformad för inomhusapplikationer som utnyttjar fördelarna med massiv MIMO och cellfri massiv MIMO. I Paper B studerar vi den extrema multiplexeringsförmågan hos RadioWeaves som kan ge höga datahastigheter med mycket låg effekt. Vi observerar att RadioWeaves-arkitekturen kan rumsligt separera användare mycket bättre än en konventionell samlokaliserad arkitektur, som 
uppväger förlusterna orsakade av gitterlober och därmed sparar mycket på sändningseffekten.

Paper C studerar synkroniseringsaspekten av distribuerad massiv MIMO. Vi föreslår ett nytt, over-the-air synkroniseringsprotokoll, som vi kallar BeamSync, för att synkronisera alla olika sändningspaneler med flera antenner. Vi visar också att strålformningen av synkroniseringssignalen i den dominerande riktningen av kanalen mellan panelerna är optimal och synkroniseringsprestandan är betydligt bättre än traditionella strålformningstekniker. 


\section{Contents}

Acknowledgements $\quad$ ix

List of Abbreviations $\quad$ xi

1 Introduction and Motivation $\quad \mathbf{1}$

1.1 Massive MIMO for 5G and Beyond . . . . . . . . . . . . . 2

1.2 Contributions of the Thesis ............... 3

1.3 Excluded Papers ................ 5

2 Collocated Massive MIMO and Distributed Massive MIMO 7

2.1 Collocated Massive MIMO . . . . . . . . . . . . . 7

2.1.1 Reciprocity ................. 8

2.1.2 Channel Models . . . . . . . . . . . . . . 8

2.1.3 Channel Hardening . . . . . . . . . . . . . . . . . 9

2.1.4 Favorable Propagation . . . . . . . . . . . . 10

2.1.5 Multi Antenna Benefits . . . . . . . . . . . . 10

2.2 Distributed Massive MIMO . . . . . . . . . . . . . . 12

2.2.1 Taxonomy .................. 13

2.2.2 System Model . . . . . . . . . . . . . . . . . 14

2.2.3 Benefits with Distributed MIMO . . . . . . . . . 15

2.2.4 Challenges in Distributed Massive MIMO . . . . . . 16

3 Random Access $\quad 19$

3.1 Massive Machine Type Communications . . . . . . . . . . 19

3.1.1 mMTC Requirements . . . . . . . . . . . . . . 19

3.1.2 MMTC Use Cases . . . . . . . . . . . . . 20

3.1.3 mMTC Traffic as Random Access . . . . . . . . . . 20

3.2 Grant Based Random Access . . . . . . . . . . . . . . . . 21

3.3 Grant-Free Random Access . . . . . . . . . . . . . . . . . . . 22

3.3.1 System Model for Massive MIMO . . . . . . . . . . . 22 
3.3.2 Compressive Sensing Approach . . . . . . . . . . . . 23

3.3 .3 Covariance Based Approach . . . . . . . . . . . . . . 24

3.4 Unsourced Random Access . . . . . . . . . . . . . . . 26

4 RadioWeaves $\quad \mathbf{2 7}$

4.1 RadioWeaves Deployment . . . . . . . . . . . . . . . . 27

4.2 Benefits with RadioWeaves . . . . . . . . . . . . 28

4.3 Deployment Constraints . . . . . . . . . . . . . . . 29

5 Conclusion and Future Work 31

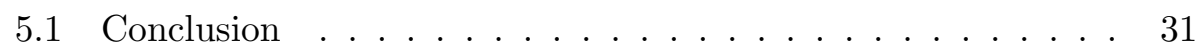

5.2 Future Works . . . . . . . . . . . . . . . . . . . 32

$\begin{array}{ll}\text { Bibliography } & 33\end{array}$

Included Papers $\quad 44$

A Clustering-based Activity Detection Algorithms 45

1 Introduction . . . . . . . . . . . . . . 47

1.1 Contributions . . . . . . . . . . . . 50

2 Signal Model And Problem Formulation . . . . . . . . . . 52

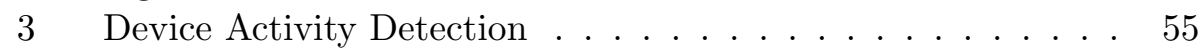

3.1 Coordinate Descent Cost Function . . . . . . . . 55

3.2 Dominant AP Based Activity Detection . . . . . . . 57

3.3 Clustering Based Activity Detection . . . . . . . . . 59

3.4 Parallel Architecture of Algorithms . . . . . . . . . . 61

3.5 Convergence of the Algorithms . . . . . . . . . . . 62

4 Simulation Results . . . . . . . . . . . . . . . 62

4.1 Simulation Model . . . . . . . . . . . . . . . 62

4.2 Results . . . . . . . . . . . . . . . . . 64

4.3 Capacity Limited Fronthauls . . . . . . . . . . . . 69

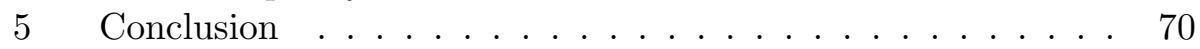

B RadioWeaves for Extreme Spatial Multiplexing $\quad 77$

1 Introduction . . . . . . . . . . . . . . 79

2 Preliminaries . . . . . . . . . . . . . . 81

$2.1 \quad$ Coordinate System . . . . . . . . . . . . . . . 81

$2.2 \quad$ Free Space Signal Propagation . . . . . . . . . . . . 81

2.3 Microstrip Patch Antenna . . . . . . . . . . . . 82 
2.4 Line of Sight Channel Model for Micro strip Patch Antenna . . . . . . . . . . . . . . 82

3 System Model and Analysis . . . . . . . . . . . . . . . 83

3.1 System Model . . . . . . . . . . . . . . . . . . . . 83

3.2 Line-of-Sight Channel Estimation . . . . . . . . . . . . 84

3.3 Power Required for Zero Forcing Operation . . . . . . 84

3.4 Downlink Spectral Efficiency . . . . . . . . . . . 85

4 Case Study . . . . . . . . . . . . . . . . . 86

5 Conclusion ...................... 90

C BeamSync: Over-The-Air Carrier Synchronization $\quad 93$

1 Introduction . . . . . . . . . . . . . . . 95

2 System Model . . . . . . . . . . . . . . . . . . 98

3 Carrier Synchronization . . . . . . . . . . . . . . . . 99

$3.1 \quad$ BeamSync Protocol . . . . . . . . . . . . . . . . 99

3.2 Optimal Beamforming Direction . . . . . . . . 100

3.3 Estimating Beamforming Direction in BeamSync . . . 102

3.4 Over-The-Air Carrier Synchronization Protocol . . . . 104

4 Simulations . . . . . . . . . . . . . . . . 105

4.1 Performance Benchmarking Schemes . . . . . . . . . 105

4.2 Results . . . . . . . . . . . . . . 106

5 Conclusion ...................... 109 


\section{Acknowledgements}

First and the foremost, I am very grateful to my supervisor, Prof. Erik G. Larsson for providing an opportunity to take up doctoral research under his guidance. In addition to Prof. Larsson, I am very grateful to my co-supervisor Prof. Emil Björnson for his guidance and encouragement. Thanks to both of you for your belief in me and supporting my Ph.D journey during the tough times and guiding me in the right direction. Your immense knowledge, expertise, patience to clear my doubts and the positive attitude helped me to grow not only as a researcher but also as a person.

Thanks to all my colleagues especially Amin, Chien, Zakir, Ziya, Ema, Özge, Giovanni, Jianan, Manoj, Kate for creating a wonderful work environment and approachable for any concerns. Special thanks to Amin for being best buddy pushing me for running and to do workouts and for the wonderful conversations over the lunch. Special thanks to Zakir and Ziya for endless technical discussions which helped me to understand concepts more deeply. Special thanks to Ema for helping me to convert the abstract into Swedish.

Thanks to my flatmate, Giacomo, for being there, trying out all my spicy foods and teaching me how to cook proper Italian pizza.

Thanks to Navya for her love and support.

Most importantly, I am very grateful to my parents, Ganesan and Sumangala and to my sister Sruthy for their love, support, care as well as to Pyarilal, my brother in law, and to our cute Mihika mol, my niece.

Unnikrishnan Kunnath Ganesan Linköping, January 2022 


\section{List of Abbreviations}

5G fifth generation of cellular network technology

AMP approximate message passing

AP access point

AWGN additive white Gaussian noise

CS compressive sensing

CSI channel state information

eMBB enhanced mobile broadband

FDD frequency-division duplex

i.i.d. independent and identically distributed

IoT internet-of-things

LoS line-of-sight

LS least-squares

MIMO multiple-input multiple-output

MMSE minimum mean-square error

mMTC massive machine-type communication

MR maximum ratio

NLoS non line-of-sight

OFDM orthogonal frequency division multiplexing

$\mathrm{RACH}$ random access channel

$\mathrm{RF} \quad$ radio frequency

SNR signal-to-noise ratio

TDD time-division duplex

URLLC ultra-reliable low-latency communication

ZF $\quad$ zero forcing 



\section{Chapter 1}

\section{Introduction and Motivation}

Over the past four decades, wireless technology has evolved drastically from being a simple telephone call to high-speed data streaming. From the beginning of a day with deep diving into social media as well as the emergence of smart homes, autonomous vehicles etc., wireless gadgets have become ingrained into our daily lives. Moreover, wireless technology has a profound impact on our social, cultural, and economic lives. The day has come where we cannot think about a day without the internet. Wireless technology acted as a catalyst in the smooth handling of operations in various sectors like business, education during the COVID-19 pandemic situation over the globe. The digital revolution enabled such a scenario possible without creating much havoc. The digital revolution made many gadgets require access to the wireless network to access data. Thus, the demand for the wireless network is not only from human-to-human interactions but also from human-to/frommachines and from machine-to-machine interactions. Also, this demand is increasing with more and more devices getting connected to the wireless network.

The future wireless networks should support three hetrogeneous services [1]: enhanced mobile broadband (eMBB), ultra reliable low latency communication (URLLC) and massive machine type communications(mMTC).

\section{eMBB}

eMBB is a natural evolution of existing $4 \mathrm{G}$ networks that provides faster data rates and a better user experience than current mobile broadband services. It is envisioned to provide a high quality of service congruous to what we enjoy from fixed broadband services. It will enable high data rate demanding applications such as real-time high definition (HD) video 
streaming, truly immersive virtual reality (VR), augmented reality (AR), etc. eMBB will provide higher capacity in densely populated areas with enhanced connectivity. Moreover, it will support high user mobility.

\section{URLLC}

URLLC provides data reliability of more than $99.999 \%$ with very low latency (of 1 millisecond) for packet transmission [2]. The use-case is designed for time-critical applications like remote surgery and remote diagnosis, smart industries to improve machine productivity, collision avoidance in intelligent transport applications etc $[3,4]$.

\section{mMTC}

mMTC is intended to support a massive number of low-energy devices with short payloads, for example, sensor data from a production industry. It focuses on internet of things (IoT) applications which require the mass deployment of billions of low-cost, low-energy sensors [5]. These devices randomly access the network and is studied under the umbrella of random access. mMTC is further detailed in Sec. 3.1.

\subsection{Massive MIMO for 5G and Beyond}

Massive multiple-input-multiple-output (MIMO) is one of the technologies which can support the envisioned three heterogenous services for $5 \mathrm{G}$ and beyond wireless networks [6]. Massive MIMO was introduced in the seminal paper [7] and since then, it has been prevalent in academic and industrial research. Massive MIMO deploys a large number of antennas at the base station and serves multiple users in the same time-frequency resource. The technology leverages the law of large numbers to gain two important properties channel hardening and favorable propagation. Moreover, with the beamforming gain from using multiple antennas, massive MIMO could improve the achievable data rates from the network.

To further increase data rates, more base stations are widely deployed in the same geographical area, which reduces the distance between the user and base station. This is commonly referred to as cell size reduction to make smaller cells and increase the data rates. However, the data rates deteriorate after some tipping point when the cell size is made very small, due to the inter-cell interference. Distributed MIMO was advocated to mitigate intercell interference and consider the interference as multiple access channels. 
Cell-free massive MIMO is the current state-of-the-art distributed MIMO technology that can mitigate inter-cell interference and operates without any concept of cell or cell boundaries. Massive MIMO technology will be further discussed in chapter 2 .

The support of heterogeneous services by collocated massive MIMO has been studied vastly in the literature. In this thesis, we study the heterogeneous services by distributed massive MIMO system. Paper A studies the random access of mMTC services in cell-free massive MIMO.

RadioWeaves technology is a new wireless infrastructure devised for indoor applications leveraging the benefits of massive MIMO and cell-free massive MIMO. In Paper B, we study the extreme multiplexing capabilities of RadioWeaves which can provide high data rates with very low power. This technology is suitable for URLLC applications as the connection can be made more reliable.

Synchronization of distributed transmit nodes is required for coherent data transmission and is a quite challenging problem. In Paper C, we study the over-the-air carrier frequency synchronization among the distributed panels of RadioWeaves.

\subsection{Contributions of the Thesis}

This thesis has three main contributions to distributed massive MIMO technology. The thesis is divided into two parts, an introduction part and a second part with three published research articles related to the contributions. The introduction section consists of an overview of massive MIMO, distributed massive MIMO, random access, RadioWeaves technology, and synchronization aspects of distributed massive MIMO.

The second part consists of three research papers that focus on different aspects of distributed massive MIMO technology. More specifically, Paper A studies grant-free random access and proposes activity detection algorithms in cell-free massive MIMO. Paper B studies the extreme multiplexing capabilities of RadioWeaves technology. Finally, Paper C proposes a carrier frequency synchronization scheme between different transceiver nodes in distributed MIMO based on beamforming. 


\section{Paper A: Clustering Based Activity Detection Algorithms for Grant-Free Random Access in Cell-Free Massive MIMO}

Authored by: Unnikrishnan Kunnath Ganesan, Emil Björnson, and Erik G. Larsson

Paper published in IEEE Transactions on Wireless Communications, vol. 69, issue 11, pp. 7520-7530, Nov. 2021.

Abstract: Future wireless networks need to support massive machine type communication (mMTC) where a massive number of devices accesses the network and massive MIMO is a promising enabling technology. Massive access schemes have been studied for co-located massive MIMO arrays. In this paper, we investigate the activity detection in grant-free random access for mMTC in cell-free massive MIMO networks using distributed arrays. Each active device transmits a non-orthogonal pilot sequence to the access points (APs) and the APs send the received signals to a central processing unit (CPU) for joint activity detection. The maximum likelihood device activity detection problem is formulated and algorithms for activity detection in cell-free massive MIMO are provided to solve it. The simulation results show that the macro diversity gain provided by the cell-free architecture improves the activity detection performance compared to co-located architecture when the coverage area is large.

\section{Paper B: RadioWeaves for Extreme Spatial Multiplexing in Indoor Environments}

Authored by: Unnikrishnan Kunnath Ganesan, Emil Björnson and Erik G. Larsson

Published in the proceedings of 2020 Asilomar Conference on Signals, Systems, and Computers

Abstract: With the advances in virtual and augmented reality, gaming applications, and entertainment, certain indoor scenarios will require vastly higher capacity than what can be delivered by $5 \mathrm{G}$. In this paper, we focus on massive MIMO for indoor environments. We provide a case study of the distributed deployment of the antenna elements over the walls of a room and not restricting the antenna separation to be half the wavelength. This is a new paradigm of massive MIMO antenna deployment known as RadioWeaves. We investigate different antenna deployment scenarios in line of sight communication. We observe that the RadioWeaves deployment can spatially separate users much better than a conventional co-located deployment, which outweighs the losses caused by grating lobes and thus 
saves a lot on transmit power. Through simulations, we show that the RadioWeaves technology can provide high rates to multiple users by spending very little power at the transmitter compared to a co-located deployment.

\section{Paper C: BeamSync: Over-The-Air Carrier Synchronization in Distributed RadioWeaves}

Authored by: Unnikrishnan Kunnath Ganesan, Sarvendranath Rimalapudi and Erik G. Larsson

Published in 25th International ITG Workshop on Smart Antennas (WSA 2021)

Abstract: In a distributed multi-antenna system, multiple geographically separated transmit nodes communicate simultaneously to a receive node. Synchronization of these nodes is essential to achieve a good performance at the receiver. RadioWeaves is a new paradigm of cell-free massive MIMO array deployment using distributed multi-antenna panels in indoor environments. In this paper, we study the carrier frequency synchronization problem in distributed RadioWeave panels. We propose a novel, over-the-air synchronization protocol, which we call as BeamSync, to synchronize all the different multi-antenna transmit panels. We also show that beamforming the synchronization signal in the dominant direction of the channel between the panels is optimal and the synchronization performance is significantly better than traditional beamforming techniques.

\subsection{Excluded Papers}

The papers mentioned in Table 1 are excluded from this thesis as them being superfluous. The paper 1 is authored by me and is a conference version of Paper A. I co-authored the paper 2, wherein a hardware architecture for grant-free random access for mMTC using coordinate descent was presented, and is based on [8] and Paper A. 
U. K. Ganesan, E. Björnson, and E. G. Larsson, "An Algorithm for 1. Grant-Free Random Access in Cell-Free Massive MIMO," in IEEE 21st International Workshop on Signal Processing Advances in Wireless Communications (SPAWC), Atlanta, GA, USA, 2020, pp. 1-5.

M. Henriksson, O. Gustafsson, U. K. Ganesan and E. G. Larsson, "An Architecture for Grant-Free Random Access Massive Machine

2. Type Communication Using Coordinate Descent," in 54th Asilomar Conference on Signals, Systems, and Computers, Pacific Grove, CA, USA, 2020, pp. 1112-1116. 


\section{Chapter 2}

\section{Collocated Massive MIMO and Distributed Massive MIMO}

Massive MIMO is the backbone physical layer technology for 5G and beyond networks to enable the services such as eMBB, URLLC and mMTC. This chapter discusses massive MIMO, both with respect to collocated and distributed sense and its benefits and the associated challenges with it.

\subsection{Collocated Massive MIMO}

Consider a single cell cellular system with a base station equipped with $M$ antennas serving $K$ single-antenna user terminals. Each antenna at the base station are equipped with its own radio frequency $(\mathrm{RF})$ chain such that the base station can digitally control the relative phase between antenna elements. This enables the base station to spatially direct the signals in any desired direction [9].

We assume the operations in a coherence block [10, Ch. 2]. Let the channel between user $k$ and the base station be $\mathbf{g}_{k} \in \mathbb{C}^{M \times 1}$, which is an $M$ dimensional complex vector. If uplink and downlink are using different frequency resources, the uplink and the downlink channel will be different. Towards this, we define reciprocity as follows. 


\subsubsection{Reciprocity}

For uplink and downlink, there are two types of transmission operation namely frequency division duplexing (FDD) and time division duplexing (TDD). In FDD, both uplink and downlink transmissions are separated in frequency and hence the uplink channel $\mathbf{g}_{k}^{\mathrm{UL}}$ and the downlink channel $\mathbf{g}_{k}^{\mathrm{DL}}$ will be different as they uses two different coherence blocks for operation. In TDD, the uplink and downlink transmissions are separated in time within a coherence block and thus, the uplink channel $\mathbf{g}_{k}^{\mathrm{UL}}$ and the downlink channel $\mathbf{g}_{k}^{\mathrm{DL}}$ will be same, i.e., $\mathbf{g}_{k}=\mathbf{g}_{k}^{\mathrm{UL}}=\mathbf{g}_{k}^{\mathrm{DL}}$. We refer to this concept as reciprocity of the channel.

FDD was a preferable mode of transmission up to $4 \mathrm{G}$ networks as the involved complexity of channel estimations is less. In massive MIMO, due to the deployment of a large number of antennas, FDD operation requires huge complexity for the channel estimation [10]. Thus, we consider TDD operation for massive MIMO and throughout this thesis, TDD operation will be assumed. With the reciprocity assumption in massive MIMO, we need to estimate the channel only in one direction and can be re-used in the other direction. Preferably, the channel is estimated in the uplink direction as channel estimation complexity in downlink increases with the increasing number of base station antennas $[9,10]$.

\subsubsection{Channel Models}

For mathematical tractability and simulations to evaluate the performance of wireless systems, various channel models are considered.

\section{Line-of-Sight (LoS) Channel Model}

A line-of-sight (LoS) channel is a simplistic channel model when the transmitter and receiver are assumed to be in free space. We assume that there is only one direct propagation path from transmitter to receiver and no scatterers in the environment. Let $P_{\mathrm{tx}}$ be the power transmitted by the transmitter. Let $r$ be the relative distance between transmitter and receiver. Then the received power at the receiver can be written as

$$
P_{\mathrm{rx}}=\frac{P_{\mathrm{tx}}}{4 \pi} G_{\mathrm{tx}} G_{\mathrm{rx}} \frac{1}{r^{2}} \frac{\lambda^{2}}{4 \pi} .
$$

where $G_{\mathrm{tx}}$ and $G_{\mathrm{rx}}$ are the directional power gains at the transmitter and receiver respectively, and $\lambda$ is the wavelength of the transmitted signal. When the directional power gains are constant, (1) gives Friis' free space 
equation [11]. The LoS channel model is also used when we have very strong LoS components compared to non LoS (NLoS) components, for example, in indoor scenarios.

\section{Uncorrelated Rayleigh Fading Channel Model}

In practical systems, when a signal is transmitted from a source, several attenuated and time-delayed copies (multi-path components) of the signal will receive at the receiver. As the propagation environment is varying, it is not feasible to track the multi-path components. Therefore, statistical modeling is considered, which will be constant for a longer time compared to the coherence time of the channel. One of the most commonly used channel models is Rayleigh fading channel model [12]. In the Rayleigh fading channel, the channel in each coherence block is modeled as

$$
\mathbf{g}_{k} \sim \mathcal{C N}(\mathbf{0}, \mathbf{C})
$$

where $\mathbf{C}$ is the covariance matrix. In uncorrelated Rayleigh fading channel model, $\mathbf{C}=\beta_{k} \mathbf{I}_{\mathrm{M}}$, where $\beta_{k}$ is the large scale fading coefficient between the user $k$ and the base station, and is a parameter depending on the distance between the user and the base station. In this thesis, we will consider uncorrelated cases only.

\subsubsection{Channel Hardening}

Consider the following expression

$$
\frac{\left\|\mathbf{g}_{k}\right\|^{2}}{\mathbb{E}\left\{\left\|\mathbf{g}_{k}\right\|^{2}\right\}} \stackrel{\text { a.s }}{\longrightarrow} 1, \text { as } M \rightarrow \infty .
$$

The expression in (3) means that the channel can be considered deterministic in asymptotic sense as $M \rightarrow \infty$. This property is known as channel hardening $[7,9,10,13]$. Thus, as we have sufficiently large number of antennas at the base station, the norm of the channel will be deterministic and this helps in simpler signal processing. Thus, in collocated massive MIMO, we deploy a large number of antennas at the base station to make use of the channel hardening property. 


\subsubsection{Favorable Propagation}

As we explained channel hardening through (3), now consider the following expression

$$
\frac{\mathbf{g}_{k}^{\mathrm{H}} \mathbf{g}_{k^{\prime}}}{\sqrt{\mathbb{E}\left\{\left\|\mathbf{g}_{k}\right\|^{2}\right\} \mathbb{E}\left\{\left\|\mathbf{g}_{k^{\prime}}\right\|^{2}\right\}}} \stackrel{a . s}{\longrightarrow} 0, \text { as } M \rightarrow \infty, k \neq k^{\prime},
$$

where $\mathbf{g}_{k}$ and $\mathbf{g}_{k^{\prime}}$ are the channel vectors for two different users. The expression in (4) means that when we have sufficiently large number of antennas at the base station, the channel between two users will be almost orthogonal and this property is known as favorable propagation $[7,9,10]$.

\subsubsection{Multi Antenna Benefits}

Channel hardening and favorable propagation along with reciprocity when operated in TDD mode, made massive MIMO a dominant and key technology for $5 \mathrm{G}$ and beyond $5 \mathrm{G}$ networks. Moreover, multiple antenna systems have other benefits which boosted the use of massive MIMO systems.

To understand further the multi-antenna benefits, consider a single-inputsingle-output (SISO) system. The signal at the receiver is given by

$$
y=g x+w,
$$

where $g$ is the channel response value, $x$ is the transmitted symbol with $\mathbb{E}\left\{\|x\|^{2}\right\} \leq \rho$, where $\rho$ is the transmitted power and $w \sim \mathcal{C N}\left(0, \sigma^{2}\right)$ is the additive white Gaussian noise (AWGN) with power $\sigma^{2}$. Let the signal-tonoise-ratio (SNR) be defined as $\frac{\rho}{\sigma^{2}}$. For a deterministic channel and assuming the channel is known at the receiver, the capacity of the SISO system [14] is

$$
C=\log _{2}\left(1+|g|^{2} \mathrm{SNR}\right) \text { bits/symbol. }
$$

Moreover, if the transmitter does not know the channel and it transmits at a rate, $R$ above the capacity of the system, an outage might occur with probability [14]

$$
\begin{aligned}
P_{\text {out }}(R) & =\operatorname{Pr}\{R>C\}=\operatorname{Pr}\left\{R>\log _{2}\left(1+|g|^{2} \mathrm{SNR}\right)\right\} \\
& =\operatorname{Pr}\left\{|g|^{2}<\frac{2^{R}-1}{\mathrm{SNR}}\right\} .
\end{aligned}
$$

For Rayleigh fading scenario, $g \sim \mathcal{C N}(0,1)$. Hence $|g|^{2}$ is exponentially distributed and thus the outage probability at high SNR is approximated as

$$
P_{\text {out }}(R) \approx \frac{2^{R}-1}{\mathrm{SNR}}
$$


From $(9), P_{\text {out }}(R) \propto \mathrm{SNR}^{-1}$. With the addition of more antennas in the system the capacity and the outage probability can be improved as explained as follows.

\section{Beamforming Gain}

Consider an uplink signal from a user to the base station equipped with $M$ antennas. This is similar to single-input-multiple-output (SIMO) scenario. The signal at the receiver, $\mathbf{y} \in \mathbb{C}^{M \times 1}$ is

$$
\mathbf{y}=\mathbf{g} x+\mathbf{w},
$$

where $\mathbf{g} \in \mathbb{C}^{M \times 1}$ is the channel response vector, $x$ is the transmitted symbol with $\mathbb{E}\left\{\|x\|^{2}\right\} \leq \rho$, where $\rho$ is the transmitted power and $\mathbf{w} \sim \mathcal{C N}\left(0, \sigma^{2} \mathbf{I}_{\mathrm{M}}\right)$ is the AWGN vector, and $\sigma^{2}$ is the noise power. Assuming the channel is known at the receiver (base station), the receiver can exploit the channel knowledge and post process the signal received as [15]

$$
\frac{\mathbf{g}^{\mathrm{H}}}{\|\mathbf{g}\|} \mathbf{y}=\|\mathbf{g}\| x+\frac{\mathbf{g}^{\mathrm{H}}}{\|\mathbf{g}\|} \mathbf{w} .
$$

The capacity of the SIMO channel is then given by [14]

$$
C=\log _{2}\left(1+\|\mathbf{g}\|^{2} \mathrm{SNR}\right) \text { bits/symbol. }
$$

The term $\|\mathbf{g}\|^{2}$ in (12) is often proportional to $M$ and hence, $\|\mathbf{g}\|^{2}$ represents an increase in the effective SNR at the receiver. Thus, this term is referred to as beamforming gain. From (12), the capacity scales logarithmically with $M$ at high SNR and linearly with $M$ at low SNR values.

The post-processing operation applied in (11) is known as the maximumratio combining (MRC) operation, which maximizes the SNR at the receiver. The same capacity, as well as beamforming gain, can be achieved in downlink as well, which is similar to a multiple-input-single-output (MISO) system, by pre-processing the transmitted signal using maximum-ratio transmission (MRT), a dual to MRC.

Thus, the capacity of a massive MIMO system significantly increases with the deployment of a large number of antennas at the base station.

\section{Spatial Diversity Gain}

Similar to SISO, system when the transmitter does not know the channel, there might occur an outage at the receiver in SIMO or MISO system. The 
outage probability for a multi antenna system is given by

$$
P_{\text {out }}(R)=\operatorname{Pr}\{R>C\}=\operatorname{Pr}\left\{\|\mathbf{g}\|^{2}<\frac{2^{R}-1}{\mathrm{SNR}}\right\} .
$$

For Rayleigh fading scenario, $\mathbf{g} \sim \mathcal{C N}\left(\mathbf{0}, \mathbf{I}_{M}\right)$ and hence, $\|\mathbf{g}\|^{2}$ is $\chi^{2}$ distributed with $M$ degrees of freedom and thus the outage probability at high SNR is approximated as

$$
P_{\text {out }}(R) \approx\left(\frac{2^{R}-1}{\mathrm{SNR}}\right)^{M} \frac{1}{M !} .
$$

From (14), $P_{\text {out }}(R) \propto \mathrm{SNR}^{-M}$. Thus the outage probability reduces with a slope $-M$ when plotted in log scale against SNR in $\mathrm{dB}$. This is referred to as spatial diversity gain with diversity order $M$.

Thus, the outage probability can be significantly reduced in a massive MIMO system with the deployment of large number antennas at the base station and the connection can be made more reliable.

To provide a uniform and high per-user data rates in future wireless networks, we need to have strong signal which is established by decreasing the distance between the users and the base station. This leads to implementation of smaller cells. As we implement smaller and smaller cells, the system capacity is limited by the inter-cell interference. Distributed systems are advocated to improve the system capacity as well as to mitigate the inter-cell interference which is explained in next section.

\subsection{Distributed Massive MIMO}

Collocated massive MIMO was shown to improve the capacity of the network and to support the ever-increasing demand, network densification is considered. The capacity increases with the dense deployment of base station access points (APs). This led to the development of microcells, picocells, and femtocells or in general small cells [16]. However, we cannot keep diminishing the cell size to exploit the capacity. At a certain point, the diminishing cell size will make the communication system perform poorly due to the increase in inter-cell interference. Thus, another technology needs to be sought to further exploit the capacity of the wireless network. This led to the development of the distributed wireless system. In a distributed massive MIMO system, a large number of service antennas or antenna panels commonly known as access points are geographically spread out in a wide area. There is no concept of cells or cell boundaries. All APs are cooperating phase 
coherently through a backhaul network. Distributed massive MIMO inherits the multiple antenna benefits of massive MIMO and provides extraordinary macro-diversity gain. Such systems can resolve inter-cell interference and can provide larger SNR as the shadowing effects will be reduced. Thus, it can provide uniformly great service by guaranteeing an excellent quality of service to all users. The advantages come at a cost of the implementation complexity of the backhaul network.

\subsubsection{Taxonomy}

Distributed wireless systems have been studied in the literature under various names with different deployment strategies. In this section, a few of them are outlined.

\section{Network MIMO}

In network MIMO [17-20], multiple cells cooperate to act as a single clustercell and serve all the users in these cells. The base stations are grouped into disjoint clusters and each cluster is served by a central processing unit (CPU). The CPU does the signal processing in a centralized fashion and is responsible for base station cooperation, collecting channel state information (CSI) from the base station for precoding/combining during downlink/uplink signaling. Network MIMO turns the interference channel into a multiple access channel and hence, can mitigate inter-cell interference.

\section{Cooperative Multi-Cell MIMO}

Conventional network MIMO relies on fully centralized signal processing and requires the exchange of instantaneous CSI among the base stations. Hence, in cooperative multi-cell MIMO [21-23], distributed signal processing with no sharing of CSI is considered to reduce the computational complexity and fronthaul requirements.

\section{CRAN}

C-RAN (Cloud Radio Access Network) [24] is a cloud computing-based architecture. The baseband processing is done at a central processor which is connected to several remote radio units(RRUs). The RRUs act as a signal forwarding device and all the processing complexity relies on the central processor. 


\section{CoMP}

CoMP(Coordinated MultiPoint) is essentially the commercial name of network/cooperative MIMO in LTE Advanced [25]. To achieve the gains envisaged with perfect channel state information in academia for CoMP, improved CSI feedback as well as improved precoding granularity was required and remained as challenging issues in practice. Thus, the theoretical and practical gap between the gains remained quite high. Even though it was standardized, it could not be implemented in practice because of different proprietary rights with different vendors which caused further challenges.

\section{Cell-Free Massive MIMO}

Cell-free massive MIMO $[16,26]$ blends in the advantages from ultra densification, collocated massive MIMO, and CoMP. It considers a massive number of cooperating APs and provides a complete cell-free architecture. The users select the APs based on the strength and those APs form a cluster to serve that specific user. Thus, the user-centric approach provides a uniform service to all the users in the network. The CSI need not be shared and can enhance local processing at the APs to provide a joint distributed signal processing. It blends in the concept of channel hardening and favorable propagation to provide simple linear signal processing and power allocation schemes to unleash the capacity of the network to its theoretical limit. Moreover, cell-free massive MIMO utilizes TDD architecture to reduce the complexity of CSI acquisition. Moreover, the excess service antennas over the number of user terminals create favorable propagation conditions with high probability [26]. In this thesis, when we say distributed massive MIMO, we refer to cell-free massive MIMO case as it blends in all the advantages of distributed topologies.

\section{RadioWeaves}

RadioWeaves is a new wireless infrastructure leveraging the benefits of massive MIMO and cell-free systems especially devised for indoor scenarios. RadioWeaves will be further discussed in detail in Chapter 4 .

\subsubsection{System Model}

We consider a distributed massive MIMO network with $M$ access points equipped with $N$ antennas serving $K$ single antenna users as shown in Fig. 1. All the $M$ APs are connected to a CPU through a backthaul. Let $\mathbf{g}_{m k}$ be the $N$-dimensional channel response vector between user $k$ and AP $m$. For 


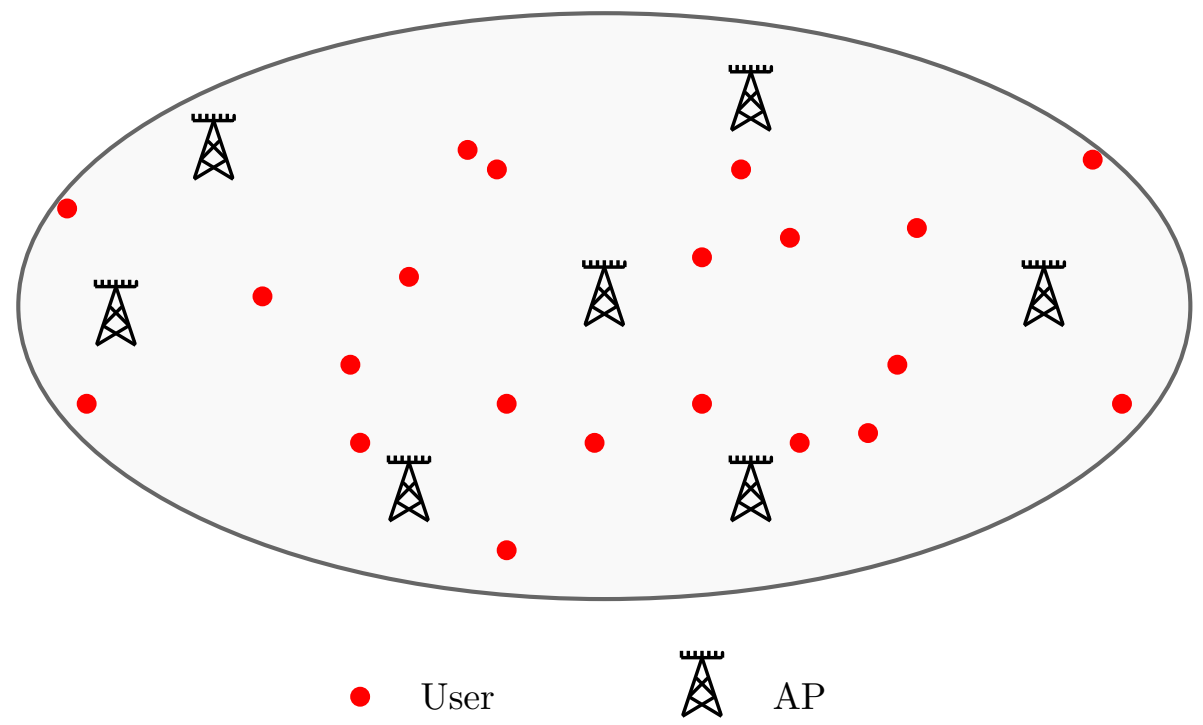

Figure 1: Distributed massive MIMO network model

an uncorrelated Rayleigh fading channel model the channel response vector is modeled as

$$
\mathbf{g}_{m k} \sim \mathcal{C N}\left(\mathbf{0}, \beta_{m k} \mathbf{I}_{N}\right)
$$

where $\beta_{m k}$ is the large scale fading coefficient between user $k$ and AP $m$. In the uplink case, the received signal $\mathbf{y}_{m}$ at AP $m$ from all the users is given by

$$
\mathbf{y}_{m}=\sqrt{\rho_{\mathrm{ul}}} \sum_{k=1}^{K} \mathbf{g}_{m k} x_{k}+\mathbf{w}_{m}
$$

where $\rho_{\mathrm{ul}}$ is the uplink power of each user, $x_{k}$ is the data from user $k$ and $\mathbf{w}_{m}$ is the noise vector. The access points either process the signal locally and sends some function of received signal to the CPU or it can send the whole signal to the CPU. In Downlink, CPU will jointly send signals to the APs and the APs will transmit the signals to the user terminals.

\subsubsection{Benefits with Distributed MIMO}

Different distributed architectures mentioned in Sec. 2.2.1 have their pros and cons. In this section, the benefits of distributed MIMO are presented which can be leveraged to design a communication system. 


\section{Macro Diversity}

In a distributed setup, the access points are distributed geographically over a wide area. This leads to ultra densification of access points near the users and every user in the network will be close to one of the APs. In a collocated scenario, the cell-edge user needs to sacrifice its performance due to large path loss. But in distributed MIMO network, this problem is avoided and every user will get uniformly better service. All the users in the distributed network will have $5-20 \mathrm{~dB}$ higher channel gain compared to collocated network [27]. This gain is known as macro-diversity gain and can make the communication link more reliable.

\section{User Centric Operation}

Distributed topology offers two types of operation, viz, network-centric and user-centric operation. In network-centric operation, a disjoint set of APs form a cluster cell and serves the users in that cluster. This operation still suffers in performance as the cluster-edge users might not be provided better service, even though they are close to APs in another cluster.

With the user-centric operation, the user picks the APs which are closest to it in terms of signal strength and those APs will be serving that specific user. This can be achieved in cell-free massive MIMO with reasonable power control policy which picks only the closest APs contributing to its service. This allows a uniform service across all users. Moreover, the network can be considered without any cells or cell boundaries.

\section{Distributed Signal Processing}

The APs can do signal processing locally instead of sending the whole received signal back to the CPU. This can avoid backhaul network cost as well as improve the latency. This helps with the APs to process the data themselves and avoids the requirement of sharing the channel state information with other APs in the serving cluster.

\subsubsection{Challenges in Distributed Massive MIMO}

Along with the implementation complexity of the backhaul network, distributed massive MIMO comes with other challenges as well. To have all the APs operate phase coherently, the APs need to be synchronized in terms of frequency and phase. Most studies related to distributed MIMO assume perfect synchronization among the distributed APs. 
To have coherent reception of the signal at the user terminal, synchronization among the distributed transmitters is required and is critical to achieve the benefits of distributed architectures. However, in practice, achieving synchronization is a challenging problem. Each transceiver in a communication system is equipped with a local oscillator circuit that generates frequency based on a reference crystal oscillator. Due to mismatches in the reference oscillator circuits, the carrier frequency generated and the start phase of the carrier signal will be different among different transceivers. Furthermore, they can drift over time for instance, due to fluctuations in temperature and voltage.

\section{Frequency Synchronization}

The carrier frequency at different transceivers will be different. This results in a carrier frequency offset between any two transceiver nodes, which degrades the performance of the communication system. In point-to-point systems, there are many available techniques to mitigate the frequency offset issues. In a global system for mobile (GSM) systems, frequency correction burst signals (FBs) sent through the frequency correction channel (FCCH) [28] are used for frequency offset estimation. For a point-to-point orthogonal frequency division multiplexing (OFDM) system, techniques studied in [29-31] are considered.

A direct extension of synchronization techniques developed for a pointto-point communication system to a distributed communication system is not feasible because the receiver observes a combined signal from different transmit nodes. A non-scalable solution to achieve carrier frequency synchronization is to provide a common carrier frequency to these distributed transmit nodes through a wired fronthaul network. This will ensure both frequency and phase synchronization at all the nodes. This relies heavily on the backhaul network and the complexity increases with an increase in the number of APs in the system.

Over-the-air carrier synchronization methods were studied in [32-34] and avoid the dependency on backhaul network. AirShare in [32] uses a dedicated emitter to transmit two low-frequency tones over the air. The distributed transceivers use a dedicated circuit to receive these tones and generate their reference signal with the difference frequency of the two tones. This technique is robust to variations in temperature and supply voltage at the emitter. However, it uses out of band frequency resources. We proposed an over-the-air carrier frequency synchronization technique using the key properties of massive MIMO namely, beamforming to further enhance the 
synchronization procedure in distributed system and is further detailed in Paper C.

\section{Phase Synchronization}

Similar to the frequency, due to mismatches in the local oscillators, the start phase of the carrier signal will also be different at different transceiver nodes. This leads to a timing mismatch with respect to global time, among the transceivers. In baseband, for a narrow band communication system, the timing mismatch manifests as a shift in the phase of the signal transmitted from different nodes at the receiver.

Let $x(t)$ be a narrowband signal with envelope $\bar{x}(t)$. Then, $x(t)$ can be represented as

$$
x(t)=\bar{x}(t) \cos \left(2 \pi f_{c} t+\phi\right),
$$

where $f_{c}$ and $\phi$ are the frequency of the carrier signal and phase offset, respectively. Considering two transmitters, which are frequency synchronized, but not phase synchronized, transmitting the same signal $\bar{x}(t)$, the superposition of the signals transmitted from both is given by

$$
\begin{aligned}
y(t) & =x_{1}(t)+x_{2}(t) \\
& =\bar{x}(t) \cos \left(2 \pi f_{c} t+\phi_{1}\right)+\bar{x}(t) \cos \left(2 \pi f_{c} t+\phi_{2}\right) \\
& =2 \bar{x}(t) \cos \left(\frac{\phi_{1}-\phi_{2}}{2}\right) \cos \left(2 \pi f_{c} t+\frac{\phi_{1}+\phi_{2}}{2}\right) .
\end{aligned}
$$

From (18), the superpositioned signal can undergo destructive interference when the phases $\phi_{1}$ and $\phi_{2}$ are not synchronized due to the term $\cos \left(\frac{\phi_{1}-\phi_{2}}{2}\right)$. Thus, in a distributed transmitting system it is very critical to estimate and correct the phase difference among the different transmitting nodes, otherwise the communication system will perform poorly. 


\section{Chapter 3}

\section{Random Access}

This chapter provides an overview of the massive machine type communications and the random access protocols. Moreover, this chapter intends to provide an idea of how massive MIMO supports random access.

\subsection{Massive Machine Type Communications}

Massive machine type communications or in short mMTC is one of the three primary use cases envisioned for future wireless networks [5,35-39]. mMTC supports extremely high connection density and focus on IoT applications deploying billions of low powered and low cost devices [40].

\subsection{1 mMTC Requirements}

MMTC use-case is characterized by following requirements and assumptions:

- Infrequent burst of data

- Low bandwidth and usage of low transmit power

- Wide area coverage

- Low mobile devices

- Long battery life upto 20 years

- Lenient latency constraints

- Support large number of users in the same time-frequency resources

- Predominantly uplink communication. 


\subsubsection{MMTC Use Cases}

The mMTC applications are designed to handle sensor networks where low energy devices with low data volume are connecting in a massive scale. Some $5 \mathrm{G}$ applications coming under this use case are

- Smart cities, smart home, smart buildings/industries [41-46]

- Intelligent agriculture systems [47]

- Patient E-Health monitoring systems [48]

- Traffic management systems.

Most applications being sensor devices, transmit data to the cloud or the server and hence, the communication is predominantly uplink. Some sensors run on batteries and might be placed in inconvenient locations and hence, changing batteries frequently is not feasible. Thus, the requirement of wireless technology is to make communication as efficient as possible with low power requirements.

\subsection{3 mMTC Traffic as Random Access}

The mMTC traffic is an infrequent burst of data and hence, the data from each device is sporadic. Thus, at any time instant, out of the whole devices in the network, only a small fraction of the users will be active. Let there be $K$ users in the network with activation probability $\epsilon \ll 1$. Let $a_{k} \in\{0,1\}$ where $a_{k}=1$ denotes that the $k^{\text {th }}$ device is active and $a_{k}=0$ that it is inactive and $\operatorname{Pr}\left\{a_{k}=1\right\}=\epsilon$ and $\operatorname{Pr}\left\{a_{k}=0\right\}=1-\epsilon$. The devices transmit data when they have data to be sent and at other times they are silent. Considering a sensor, it needs to send data when an event has occurred. These events will be random and hence the devices will be accessing the network randomly. Thus, the network should be able to handle the random access requests from a massive number of devices. Massive MIMO was shown to be a promising technology to support massive access by exploiting the spatial degrees of freedom available in the network to let many users transmit simultaneously $[7,36,49]$. Two types of random access have been proposed in the massive MIMO literature, viz, grant-based and grant-free random access schemes. They are discussed further in the next sections. 


\subsection{Grant Based Random Access}

Grant-based random access protocol uses a physical random access channel (PRACH) [50,51], where each active device randomly picks a preamble sequence from a shared pool of orthogonal sequences and uses the selected sequence to inform the base station that it has data to transmit. In wireless systems, the channel coherence interval is limited and hence, the set of orthogonal preamble sequences is finite. The preamble does not carry any information or data and is a robust entity to gain synchronization with the base station and plays a role of a pilot sequence. The devices are working independently and hence, they select the preamble sequence in an uncoordinated way, thereby multiple devices end up picking the same preamble. Collisions occur at the base station when the same preamble is picked by multiple devices. The base station checks whether a preamble is active or not [52] and sends a random access response corresponding to active preambles, to convey the physical resource allocation. Each device upon receiving the response from the base station sends a radio resource control (RRC) connection request to obtain physical resources for data transmission. If more than one device has picked the same preamble, they will request for same resources and the base station will detect a collision. Base station need to resolve the contention and can involve multiple steps to resolve the collisions. This increases the latency of the communication.

Grant-based massive random access schemes are studied in [52-58]. A strongest user collision resolution (SUCR) technique was proposed in [55] for a massive MIMO scenario that exploits the channel hardening features. In SUCR protocol, each user can detect collision and determine how strong the contender's channel is and transmit back to the base station only if it has the strongest channel gain. A scheme where devices can contend for idle preamble sequences along with SUCR protocol is proposed in [57]. The authors in [58] study about the success probability of random access protocol.

Grant-based random access protocols permit simple signal processing at the base station. As the traffic is sporadic in mMTC, there is a high risk of collision of potentially active users. Thus, the probability of the multiple active devices selecting the same preamble sequence is quite high. Hence, the average latency involved in the grant-based random access protocol is quite high due to collision resolution. Moreover, the time spend for collision resolution or the signaling overhead to achieve complete communication is quite high compared to the short payload data mMTC devices carry. Considering the average latency incurred and signaling overhead requirements, it is inefficient to use the conventional grant-based random access methods 


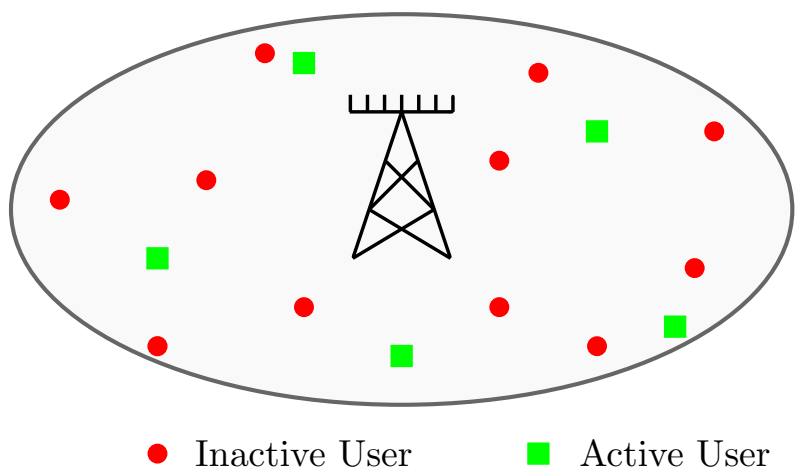

Figure 2: Network Model for mMTC.

for mMTC. In [59], several consecutive preambles are combined to form a super preamble to improve the success rate of random access and to obtain a $\mathrm{RACH}-$ based grant-free type random access.

\subsection{Grant-Free Random Access}

To overcome the limitations of grant-based random access schemes, various grant-free protocols [60] have been proposed for the active devices to access the wireless network without a grant, out of which RACH-less grant-free random access has gained some quite attention in massive MIMO literature. In this thesis, we will focus more on RACH-less grant-free random access and in short, we will refer to it as simply grant-free random access. The grant-free approach reduces the access latency and the signaling overhead compared to RACH-based approaches at the expense of sophisticated signal processing at the base station. In the grant-free random access scheme, each device is assigned a unique pilot sequence and the active devices access the network using this sequence. Thus, the approach is non-orthogonal multiple access (NOMA) scheme. Various approaches are studied in the literature for grant-free random access and are outlined in upcoming subsections.

\subsubsection{System Model for Massive MIMO}

Consider a single cell network with a base station equipped with $M$ antennas serving $K$ single antenna users as shown in Fig. 2. Due to the sporadic nature of the traffic of mMTC devices, only a small fraction of the $K$ users are active at any given time instant and each device transmits independently with an 
activation probability $\epsilon \ll 1$. Let $a_{k} \in\{0,1\}$ denote the whether device is active or not. Let $\mathbf{a}=\left(a_{1}, a_{2}, \cdots, a_{K}\right)$ denote the activity of $K$ users at any time instant. Let $\tau_{c}$ be the number of channel uses per coherence interval. Due to the large number of users, typically $K \gg \tau_{c}$, assigning orthogonal pilot sequences to each user is not feasible. Instead, we assign non-orthogonal unique signature sequence, $\mathbf{s}_{k} \in \mathbb{C}^{L \times 1}$ to each user $k$, where $L \leq \tau_{c}$ is the signature sequence length. We assume that the signature sequences of all the users are known at the base station.

The signal $\mathbf{y}_{m} \in \mathbb{C}^{L \times 1}$ received at the $m^{\text {th }}$ antenna of the base station is given by

$$
\begin{aligned}
\mathbf{y}_{m} & =\sum_{k=1}^{K} a_{k} \rho_{k}^{\frac{1}{2}} g_{m k} \mathbf{s}_{k}+\mathbf{w}_{m} \\
& =\mathbf{S D}_{\mathbf{a}} \mathbf{D}_{\boldsymbol{\rho}}^{\frac{1}{2}} \mathbf{g}_{m}+\mathbf{w}_{m},
\end{aligned}
$$

where $\mathbf{S}=\left[\begin{array}{llll}\mathbf{s}_{1} & \mathbf{s}_{2} & \ldots & \mathbf{s}_{K}\end{array}\right] \in \mathbb{C}^{L \times K}$ is the collection of all signature sequences, $\rho_{k}$ is the power transmitted by user $k, \mathbf{D}_{\mathbf{a}}=\operatorname{diag}(\mathbf{a}), \mathbf{D}_{\rho}=$ $\operatorname{diag}\left(\rho_{1}, \rho_{2}, \ldots, \rho_{K}\right), \mathbf{g}_{m}=\left[\begin{array}{llll}g_{m 1} & g_{m 2} & \ldots & g_{m K}\end{array}\right]^{\mathrm{T}} \in \mathbb{C}^{K \times 1}$ is the channel response vector from all $K$ users to the $m^{t h}$ antenna of the base station and $\mathbf{w}_{m} \sim \mathcal{C N}\left(\mathbf{0}, \sigma^{2} \mathbf{I}_{L}\right)$ is the independent AWGN vector.

Thus, the overall signal received at the base station $\mathbf{Y} \in \mathbb{C}^{L \times M}$ can be expressed as

$$
\mathbf{Y}=\mathbf{S D}_{\mathbf{a}} \mathbf{D}_{\rho}^{\frac{1}{2}} \mathbf{G}+\mathbf{W}
$$

where $\mathbf{G}=\left[\begin{array}{llll}\mathbf{g}_{1} & \mathbf{g}_{2} & \ldots & \mathbf{g}_{M}\end{array}\right] \in \mathbb{C}^{K \times M}$ is the channel matrix between the $K$ users and base station and $\mathbf{W}=\left[\begin{array}{llll}\mathbf{w}_{1} & \mathbf{w}_{2} & \ldots & \mathbf{w}_{M}\end{array}\right] \in \mathbb{C}^{L \times M}$ is the noise matrix.

\subsubsection{Compressive Sensing Approach}

The sparse nature of the device activity pattern $\mathbf{a}$, helps to formulate the activity detection problem in (20) as a compressive sensing (CS) problem [61, 62 ] and greedy pursuit algorithms were leveraged to solve it [63-65], with the assumption that the number of active users is known a priori.

Bayesian inference based algorithms like approximate message passing (AMP) are utilized in [66-71] which are computationally efficient for activity detection. To further discuss the AMP algorithm, consider all the devices are transmitting at the same power $\rho$. Then (20) can be written as

$$
\mathbf{Y}=\sqrt{\rho} \mathbf{S D}_{\mathbf{a}} \mathbf{G}+\mathbf{W} .
$$


Let $\mathbf{x}_{k}=a_{k} \mathbf{g}_{k}$. Define

$$
\mathbf{X}=\left[\begin{array}{llll}
\mathbf{x}_{1} & \mathbf{x}_{2} \cdots \mathbf{x}_{K}
\end{array}\right]
$$

Then (23) can be rewritten as

$$
\mathbf{Y}=\sqrt{\rho} \mathbf{S X}+\mathbf{W} .
$$

The rows of the matrix $\mathbf{X}$ follows a Bernoulli Gaussian distribution

$$
p_{\mathbf{x}_{k}}=(1-\epsilon) \delta_{0}+\epsilon p_{\mathbf{g}_{k}}, \forall k .
$$

The rows of $\mathbf{X}$ has many zeros and is thus, matrix $\mathbf{X}$ is row sparse and the reconstruction problem is a CS problem. The reconstruction can be done using AMP algorithm.

\section{AMP Algorithm}

The message passing algorithm was proposed in [72] and vector AMP algorithm was proposed in [73]. Assuming prior distribution on channel and (24), the AMP algorithm tries to minimize the mean squared error (MSE) on the estimate $\hat{\mathbf{X}}$ of $\mathbf{X}$ based on observations $\mathbf{Y}$

$$
\mathrm{MSE}=\mathbb{E}\left\{\|\hat{\mathbf{X}}(\mathbf{Y})-\mathbf{X}\|^{2}\right\} .
$$

Starting with $\mathbf{X}^{0}=\mathbf{0}$ and $\mathbf{R}^{0}=\mathbf{Y}$, the AMP algorithm proceeds in each iteration as $[66,67,73]$

$$
\begin{aligned}
\mathbf{x}_{k}^{t+1} & =\eta_{t, k}\left(\left(\mathbf{R}^{t}\right)^{\mathrm{H}} \mathbf{s}_{k}+\mathbf{x}_{k}^{t+1}\right) \\
\mathbf{R}^{t+1} & =\mathbf{Y}-\mathbf{S} \mathbf{X}^{t+1}+\frac{K}{L} \mathbf{R}^{t} \sum_{k=1}^{K} \frac{\eta_{t, k}^{\prime}\left(\left(\mathbf{R}^{t}\right)^{\mathrm{H}} \mathbf{s}_{k}+\mathbf{x}_{k}^{t+1}\right)}{K}
\end{aligned}
$$

where $\mathbf{X}^{t}=\left[\begin{array}{llll}\mathbf{x}_{1}^{t} & \mathbf{x}_{2}^{t} & \cdots & \mathbf{x}_{K}^{t}\end{array}\right]^{\mathrm{T}}$ is the estimate of $\mathbf{X}$ at iteration $t$ and $\mathbf{R}^{t}$ is the residual. The function $\eta_{t, k}(\cdot)$ is denoiser function.

The CS based AMP algorithms perform better when the number of active devices is smaller than the pilot sequence length [69,72] and will degrade severely otherwise.

\subsubsection{Covariance Based Approach}

The activity detection probelm in (20) can be considered as a maximum likelihood (ML) detection problem [8,74-78]. Assuming uncorrelated channel fading across the antennas of the base station, the channel response vector 
for user $k$ is given by $\mathbf{g}_{k} \sim \mathcal{C N}\left(\mathbf{0}, \beta_{\mathbf{k}} \mathbf{I}_{\mathbf{M}}\right)$. Then the observations at all antennas are independent and each column of matrix $\mathbf{Y}$ is distributed as $\mathbf{y}_{m} \sim \mathcal{C N}(\mathbf{0}, \mathbf{Q}), \forall m$, where $\mathbf{Q}$ is the covariance matrix given by

$$
\mathbf{Q}=\mathbf{S D}_{\gamma} \mathbf{S}^{\mathrm{H}}+\sigma^{2} \mathbf{I}_{L}
$$

where $\mathbf{D}_{\boldsymbol{\gamma}}=\operatorname{diag}\{\boldsymbol{\gamma}\}=\operatorname{diag}\left\{\gamma_{1}, \gamma_{2}, \cdots, \gamma_{K}\right\}$ and $\gamma_{k}=a_{k} \beta_{k} \rho_{k}$. The vector $\gamma$ is a sparse vector consisting of power levels of active devices and zeros for inactive devices. For a given $\boldsymbol{\gamma}$, the likelihood of $\mathbf{Y}$ is given by

$$
\begin{aligned}
P(\mathbf{Y} \mid \gamma) & =\prod_{m=1}^{M} \frac{1}{|\pi \mathbf{Q}|} \exp \left(-\mathbf{y}_{m} \mathbf{Q}^{-1} \mathbf{y}_{m}\right) \\
& =\frac{1}{|\pi \mathbf{Q}|^{M}} \exp \left(-\operatorname{Tr}\left\{\mathbf{Q}^{-1} \mathbf{Y} \mathbf{Y}^{\mathrm{H}}\right\}\right)
\end{aligned}
$$

Thus, the ML estimate of $\gamma$ is

$$
\hat{\boldsymbol{\gamma}}=\underset{\boldsymbol{\gamma} \geq \mathbf{0}}{\operatorname{argmin}} \log (\mathbf{Q})+\operatorname{Tr}\left\{\mathbf{Q}^{-1} \frac{\mathbf{Y} \mathbf{Y}^{\mathrm{H}}}{M}\right\} .
$$

The optimization problem in (30) is not convex as it is a sum of concave and convex functions in $\gamma$ [79]. However, if the convex cone generated by the pilot sequences defined by $\sum_{k=1}^{K} \beta_{k} \mathbf{s}_{k} \mathbf{s}_{k}^{\mathrm{H}}$ coincides with the $L \times L$ positive semi definite (PSD) matrices, then the ML optimization problem have global minimizers [75, Th. 2] and can be solved using coordinate descend approach $[8,74,76]$.

The covariance-based approach outperforms CS based approaches for activity detection and can support more number of devices. Joint activity and data detection is proposed in [8] and the performance analysis of the covariance-based approach is provided showing the superiority over the AMP based approach by exploiting the asymptotic properties of maximum likelihood estimator. The covariance based method makes better use of the multiple antennas to improve detection accuracy compared to AMP based CS approaches. Asynchronous mMTC was considered in [80]. Activity detection in multi-cell massive MIMO has been considered in [81,82]. Activity detection in cell-free massive MIMO systems with AMP approach is studied in [83]. We in Paper A considered covariance based approach for activity detection for cell-free massive MIMO.

Deep learning based activity detection approaches are considered in [8486]. 


\subsection{Unsourced Random Access}

In many IoT applications, it might be of interest to only send the messages and not their identity. Those applications employ a common codebook and when it has a message to send, it picks the corresponding message from the codebook and sends it to the base station. The base station decodes the list of messages from the active users. This can be a typical scenario in a production industry where the sensors just need to send the data to the server. Many sensors will be used for the same purpose and will be geographically located at different locations. Thus, they have the same messages (measurements) to send when similar events occur near them. If the user wants to reveal their identity, it can embed its ID in the message. This poses a problem known as unsourced random access and was first posed in [87].

Authors in $[88,89]$, proposed a coding scheme for the unsourced scenario with real adder AWGN channel and posed the problem as a classical sparse support identification problem which is well studied in CS literature. The AMP approach was used in [90] and covariance-based coordinate descend approach was used in $[77,91]$ for unsourced random access scenarios. 


\section{Chapter 4}

\section{RadioWeaves}

RadioWeaves technology is a new wireless access infrastructure proposed in [92], in which a fabric of distributed electronic circuits consisting of the radio and computing resources serve as a massive distributed antenna array. The fabric will get weaved into conventional buildings and objects and thus, the RadioWeaves will be invisibly embedded in the environment. This helps to scale up the wireless services in a non-intrusive way. The RadioWeaves technology is built upon the concepts of massive MIMO and leverages the ideas of cell-free wireless access and large intelligent surfaces. It brings the access and the intelligence close to the devices and thus, offers superior coverage and reduces the power consumption. The technology is envisioned to have unprecedented capabilities like service connectivity, energy efficiency, reliability, low latency, etc.

\subsection{RadioWeaves Deployment}

RadioWeaves technology deploys the panel of electromagnetic elements and the computing resources in a distributed manner. The technology operates on a cell-free networking approach and is designed to a create favorable propagation environment for users.

A conventional cellular network considers many users surrounding a base station and the system needs to spend huge transmit power. The cell-free approach changes the situation around by deploying a large number of distributed antennas and make the users surrounded by a large number of access point antennas. This helps to use relatively low power compared to the cellular network. RadioWeaves technology leverages on this idea of bringing a large number of access point antennas very close to the users. Moreover, 
dense deployment of access point antennas results in a significant reduction in the average distance between the user and closest antennas. It can thus, create a direct line of sight channel environment which can further enhance the link budget.

Practically, in massive MIMO, the energy predominantly comes from some specific directions and is quite far from usually assumed i.i.d. Rayleigh scenarios. Moreover, practical antennas exhibit directional gain patterns. In RadioWeaves deployment, one can allocate resources and adjust the antenna orientation and positioning to create a good link budget scenario based on high energy directions and the directional gain of practical antennas can be used to promote the desired directions.

\subsection{Benefits with RadioWeaves}

There are many benefits with deploying RadioWeaves technology. In this section, I outline certain benefits envisioned with RadioWeaves technology.

\section{Increased Spatial Diversity}

The RadioWeaves topology is designed to maximize the probability of a terminal being close to at least a handful of antennas and in a non/shadowed and favorite direction with respect to the arrays. This provides favorable propagation conditions yielding a superior degree of macro-diversity against signal blockage. Moreover, the angular directions from a terminal to the service antennas span a broad range. The phase-aligned transmission towards the terminal results in signal power strongly concentrated in a small region around the user, creating favorable propagation conditions for the transmission of multiple data streams. Thus, RadioWeaves can improve coverage, reduce power consumption and increase system capacity. A case study regarding the reduction in power consumption and improved system capacity is done in Paper B.

\section{Ultra Robust Operation}

URLLC is an envisioned use case for future wireless networks with real-time systems requiring imperceptible latency at the application level with high reliability. Implementing a connect-compute fabric that can run applications near the terminals can meet the latency requirements. Also, the dispersed wireless access structure and the directed antenna arrays can ensure more robust coverage to time-critical applications. 


\section{Energy and Bandwidth Efficiency}

The proximity of access points and extreme multiplexing capabilities can reduce the power consumption of user terminals at the expense of local compute engines. Also, computing locally in RadioWeaves improves the bandwidth efficiency whereas the central cloud computing networks run into bandwidth bottlenecks. The distributed architecture in RadioWeaves with phase-aligned transmission helps in generating a strong energy field in the favorable field of view of certain devices thereby exciting them to generate energy. This helps in wireless power transfer to devices and can bring down global energy consumption. In combination, RadioWeaves can reduce power consumption by larger orders of magnitude. The energy efficiency of RadioWeaves helps in supporting mMTC.

\section{Reliable Positioning}

Applications like autonomous navigating robots, location-aware IoT services envisioned in beyond $5 \mathrm{G}$ networks pose requirements on reliable positioning beyond the current capabilities. A centralized cellular system is not well suited to determine the positions of terminals within its coverage reliably. RadioWeaves with its distributed implementation increases the probability of line-of-sight and also, with the diverse location of antenna arrays helps to reduce outliers. Thus, RadioWeaves provides reliable positioning capabilities.

\subsection{Deployment Constraints}

The physics for wireless signal propagation says the fact that the path loss increases with distance and decreases the signal strength. Thus large power is required to combat the path loss in wireless environments. The main idea behind RadioWeaves is to bring the access points close to the users, therefore, achieve better performance with lower power requirements. A major engineering effort is needed to install the access points close to the user. Simple and easily installable technology is proposed in $[27,93]$ wherein the access points are deployed as Radio Stripes. With Radio Stripes, the radio access points with computing capabilities are inbuilt on a strip that can be easily mounted on any surface.

In a distributed architecture, a major bottleneck is created by the interconnection among the access points. The delays occurring between the connections between array panels affect the coherent processing. Dedicated calibration and synchronization solutions are required for the efficient work- 
ing of RadioWeaves technology. In Paper C, I studied the carrier frequency synchronization aspects in RadioWeaves and proposed a scheme called as BeamSync for faster and efficient frequency synchronization. 


\section{Chapter 5}

\section{Conclusion and Future Work}

In this chapter, I briefly conclude what is achieved through this thesis and some future ongoing works. Detailed conclusions about the research works are presented in the included papers.

\subsection{Conclusion}

In the first part of this thesis, an overview of the massive MIMO and the distributed massive MIMO, random access in massive MIMO, and RadioWeaves technology are presented. In the second part, three different research aspects of distributed MIMO, viz, random access, extreme multiplexing, and synchronization are studied and presented.

Paper A studied random access in the cell-free scenario and proposed low complexity algorithms based on covariance-based approach for activity detection. It was shown that, with macro-diversity gain from cell-free systems, the network can provide superior performance for activity detection compared to a collocated network.

RadioWeaves is a new wireless infrastructure devised especially for indoor scenarios leveraging the advantages of massive MIMO and distributed systems. Paper B studied the extreme multiplexing capabilities of RadioWeaves technology. RadioWeaves technology can provide high data rates by spending little transmit power to many users. Moreover, it was shown that the loss from the grating lobes which occurs by spreading out the antennas, can be overcome by the spatial resolution provided by RadioWeaves technology.

In Paper $\mathrm{C}$, the carrier frequency synchronization among the distributed radio panels in the RadioWeaves system is studied. An algorithm for frequency synchronization over-the-air based on fully digital beamforming capabilities 
of massive MIMO is proposed. The proposed algorithm was shown to have significant performance improvement compared to traditional analog beamforming techniques (fixed grid-based schemes) and the performance sharply increases with an increasing number of antennas deployed at each RadioWeaves panel.

\subsection{Future Works}

Massive MIMO is considered the promising technology for future wireless networks. The research community is looking into various aspects of massive MIMO to unleash its complete potential to bring better services to the community. Towards this, some works are ongoing as follows

- Study about the phase synchronization in distributed MIMO and improve the estimate of phase for coherent distributed processing.

- Massive MIMO for digital divide: Study the true potential of massive MIMO with long-distance communication.

- Through a framework to evaluate the link layer performance of RadioWeaves technology, study the different deployment strategies. 


\section{Bibliography}

[1] A. Osseiran, F. Boccardi, V. Braun, K. Kusume, P. Marsch, M. Maternia, O. Queseth, M. Schellmann, H. Schotten, H. Taoka et al., "Scenarios for $5 \mathrm{G}$ mobile and wireless communications: the vision of the METIS project," IEEE communications magazine, vol. 52, no. 5, pp. 26-35, 2014.

[2] Z. Li, M. A. Uusitalo, H. Shariatmadari, and B. Singh, "5G URLLC: Design challenges and system concepts," in 2018 15th International Symposium on Wireless Communication Systems (ISWCS). IEEE, 2018, pp. 1-6.

[3] H. Chen, R. Abbas, P. Cheng, M. Shirvanimoghaddam, W. Hardjawana, W. Bao, Y. Li, and B. Vucetic, "Ultra-reliable low latency cellular networks: Use cases, challenges and approaches," IEEE Communications Magazine, vol. 56, no. 12, pp. 119-125, 2018.

[4] K. S. Kim, D. K. Kim, C.-B. Chae, S. Choi, Y.-C. Ko, J. Kim, Y.-G. Lim, M. Yang, S. Kim, B. Lim et al., "Ultrareliable and low-latency communication techniques for tactile internet services," Proceedings of the IEEE, vol. 107, no. 2, pp. 376-393, 2018.

[5] C. Bockelmann, N. Pratas, H. Nikopour, K. Au, T. Svensson, Č. Stefanović, P. Popovski, and A. Dekorsy, "Massive machine-type communications in 5G: Physical and MAC-layer solutions," IEEE Communications Magazine, vol. 54, no. 9, pp. 59-65, 2016.

[6] F. Boccardi, R. W. Heath, A. Lozano, T. L. Marzetta, and P. Popovski, "Five disruptive technology directions for 5G," IEEE communications magazine, vol. 52, no. 2, pp. 74-80, 2014.

[7] T. L. Marzetta, "Noncooperative cellular wireless with unlimited numbers of base station antennas," IEEE Trans. on Wireless Commun., vol. 9, no. 11 , pp. 3590-3600, 2010.

[8] Z. Chen, F. Sohrabi, Y.-F. Liu, and W. Yu, "Covariance based joint activity and data detection for massive random access with massive MIMO," in IEEE Int. Conf. on Commun. (ICC), 2019, pp. 1-6.

[9] E. Björnson, J. Hoydis, and L. Sanguinetti, "Massive mimo networks: Spectral, energy, and hardware efficiency," Foundations and Trends in Signal Processing, vol. 11, no. 3-4, pp. 154-655, 2017. 
[10] T. L. Marzetta, E. G. Larsson, H. Yang, and H. Q. Ngo, Fundamentals of Massive MIMO. Cambridge University Press, 2016.

[11] H. T. Friis, "A note on a simple transmission formula," Proceedings of the IRE, vol. 34, no. 5, pp. 254-256, 1946.

[12] T. S. Rappaport et al., Wireless communications: principles and practice. Prentice Hall PTR New Jersey, 1996, vol. 2.

[13] R. W. Heath Jr and A. Lozano, Foundations of MIMO communication. Cambridge University Press, 2018.

[14] D. Tse and P. Viswanath, Fundamentals of wireless communication. Cambridge university press, 2005.

[15] E. Telatar, "Capacity of multi-antenna Gaussian channels," European Transactions on Telecommunications, vol. 10, no. 6, pp. 585-595, 1999.

[16] H. Q. Ngo, A. Ashikhmin, H. Yang, E. G. Larsson, and T. L. Marzetta, "Cell-free massive MIMO versus small cells," IEEE Trans. on Wireless Commun., vol. 16, no. 3, pp. 1834-1850, 2017.

[17] G. Foschini, "The value of coherent base station coordination," in Conference on Information Sciences and Systems (CISS), Johns Hopkins University, Mar. 2005, 2005.

[18] M. K. Karakayali, G. J. Foschini, and R. A. Valenzuela, "Network coordination for spectrally efficient communications in cellular systems," IEEE Wireless Communications, vol. 13, no. 4, pp. 56-61, 2006.

[19] S. Venkatesan, A. Lozano, and R. Valenzuela, "Network MIMO: Overcoming intercell interference in indoor wireless systems," in 2007 Conference Record of the Forty-First Asilomar Conference on Signals, Systems and Computers. IEEE, 2007, pp. 83-87.

[20] G. Caire, S. A. Ramprashad, and H. C. Papadopoulos, "Rethinking network MIMO: Cost of CSIT, performance analysis, and architecture comparisons," in 2010 Information Theory and Applications Workshop (ITA). IEEE, 2010, pp. 1-10.

[21] D. Gesbert, S. Hanly, H. Huang, S. S. Shitz, O. Simeone, and W. Yu, "Multi-cell MIMO cooperative networks: A new look at interference," IEEE journal on selected areas in communications, vol. 28, no. 9, pp. 1380-1408, 2010. 
[22] A. Papadogiannis, D. Gesbert, and E. Hardouin, "A dynamic clustering approach in wireless networks with multi-cell cooperative processing," in 2008 IEEE International Conference on Communications. IEEE, 2008, pp. 4033-4037.

[23] E. Björnson, R. Zakhour, D. Gesbert, and B. Ottersten, "Cooperative multicell precoding: Rate region characterization and distributed strategies with instantaneous and statistical CSI," IEEE Transactions on Signal Processing, vol. 58, no. 8, pp. 4298-4310, 2010.

[24] "C-RAN white paper: The road towards green RAN," 2014. [Online]. Available: http://labs.chinamobile.com/cran

[25] "Coordinated Multi-Point Operation for LTE Physical Layer Aspects," 3GPP, (Release 11) Version 11.1.0, 3GPP TR 36.819, Tech. Rep., Dec. 1998.

[26] Ö. T. Demir, E. Björnson, and L. Sanguinetti, "Foundations of usercentric cell-free massive MIMO," Foundations and Trends@ in Signal Processing, vol. 14, no. 3-4, pp. 162-472, 2021.

[27] G. Interdonato, E. Björnson, H. Q. Ngo, P. Frenger, and E. G. Larsson, "Ubiquitous cell-free massive MIMO communications," EURASIP Journal on Wireless Communications and Networking, vol. 2019, no. 1, p. 197, 2019.

[28] GSM Recommendation 05.01: Physical Layer on Radio Path. ETSI, 1998.

[29] P. H. Moose, "A technique for orthogonal frequency division multiplexing frequency offset correction," IEEE Transactions on Communications, vol. 42, no. 10, pp. 2908-2914, Oct. 1994.

[30] T. M. Schmidl and D. C. Cox, "Robust frequency and timing synchronization for OFDM," IEEE Transactions on Communications, vol. 45, no. 12, pp. 1613-1621, Dec. 1997.

[31] D. Huang and K. B. Letaief, "Carrier frequency offset estimation for OFDM systems using null subcarriers," IEEE Transactions on Communications, vol. 54, no. 5, pp. 813-823, May 2006.

[32] O. Abari, H. Rahul, D. Katabi, and M. Pant, "Airshare: Distributed coherent transmission made seamless," in IEEE Conference on Computer Communications (INFOCOM). IEEE, 2015, pp. 1742-1750. 
[33] H. V. Balan, R. Rogalin, A. Michaloliakos, K. Psounis, and G. Caire, "AirSync: Enabling distributed multiuser MIMO with full spatial multiplexing," IEEE/ACM Transactions on Networking, vol. 21, no. 6, pp. 1681-1695, Dec. 2013.

[34] R. Rogalin, O. Y. Bursalioglu, H. Papadopoulos, G. Caire, A. F. Molisch, A. Michaloliakos, V. Balan, and K. Psounis, "Scalable synchronization and reciprocity calibration for distributed multiuser MIMO," IEEE Transactions on Wireless Communications, vol. 13, no. 4, pp. 1815-1831, Apr. 2014.

[35] E. Dutkiewicz, X. Costa-Perez, I. Z. Kovacs, and M. Mueck, "Massive machine-type communications," IEEE Network, vol. 31, no. 6, pp. 6-7, 2017.

[36] A.-S. Bana, E. De Carvalho, B. Soret, T. Abrão, J. C. Marinello, E. G. Larsson, and P. Popovski, "Massive MIMO for Internet of Things (IoT) Connectivity," Physical Communication, vol. 37, pp. 1-17, 2019.

[37] E. G. Larsson, O. Edfors, F. Tufvesson, and T. L. Marzetta, "Massive MIMO for next generation wireless systems," IEEE Communications Magazine, vol. 52, no. 2, pp. 186-195, 2014.

[38] A. Biral, M. Centenaro, A. Zanella, L. Vangelista, and M. Zorzi, "The challenges of M2M massive access in wireless cellular networks," Digital Communications and Networks, vol. 1, no. 1, pp. 1-19, 2015.

[39] A. Zanella, M. Zorzi, A. F. dos Santos, P. Popovski, N. Pratas, Č. Stefanović, A. Dekorsy, C. Bockelmann, B. Busropan, and T. A. Norp, "M2M massive wireless access: challenges, research issues, and ways forward," in 2013 IEEE Globecom Workshops (GC Wkshps). IEEE, 2013, pp. 151-156.

[40] Ericsson Mobility Report. Ericsson, June 2019.

[41] C. Perera, A. Zaslavsky, P. Christen, and D. Georgakopoulos, "Context aware computing for the internet of things: A survey," IEEE communications surveys \& tutorials, vol. 16, no. 1, pp. 414-454, 2013.

[42] S. Andreev, O. Galinina, A. Pyattaev, M. Gerasimenko, T. Tirronen, J. Torsner, J. Sachs, M. Dohler, and Y. Koucheryavy, "Understanding the IoT connectivity landscape: a contemporary M2M radio technology roadmap," IEEE Communications Magazine, vol. 53, no. 9, pp. 32-40, 2015. 
[43] A. Zanella, N. Bui, A. Castellani, L. Vangelista, and M. Zorzi, "Internet of things for smart cities," IEEE Internet of Things journal, vol. 1, no. 1, pp. 22-32, 2014.

[44] Y. Mehmood, F. Ahmad, I. Yaqoob, A. Adnane, M. Imran, and S. Guizani, "Internet-of-things-based smart cities: Recent advances and challenges," IEEE Communications Magazine, vol. 55, no. 9, pp. 16-24, 2017.

[45] H. Han, J. Zhao, W. Zhai, Z. Xiong, and W. Lu, "Smart City Enabled by 5G/6G Networks: An Intelligent Hybrid Random Access Scheme," arXiv preprint arXiv:2101.06421, 2021.

[46] L. Da Xu, W. He, and S. Li, "Internet of things in industries: A survey," IEEE Transactions on industrial informatics, vol. 10, no. 4, pp. 22332243, 2014.

[47] N. Javaid, A. Sher, H. Nasir, and N. Guizani, "Intelligence in IoT-Based 5G Networks: Opportunities and Challenges," IEEE Communications Magazine, vol. 56, no. 10, pp. 94-100, 2018.

[48] B. Wang, Y. Sun, C. Yuan, and X. Xu, "LESLA: A smart solution for SDN-enabled mMTC E-health monitoring system," in Proceedings of the 8th ACM MobiHoc 2018 Workshop on Pervasive Wireless Healthcare Workshop, 2018, pp. 1-6.

[49] E. De Carvalho, E. Bjornson, J. H. Sorensen, P. Popovski, and E. G. Larsson, "Random access protocols for massive MIMO," IEEE Communications Magazine, vol. 55, no. 5, pp. 216-222, 2017.

[50] 3GPP, Radio Resource Control (RRC). 3rd Generation Partnership Project (3GPP), TS TS-36331, Aug. 2010.

[51] G. C. Madueno, Č. Stefanović, and P. Popovski, "Efficient LTE access with collision resolution for massive M2M communications," in 2014 IEEE Globecom Workshops (GC Wkshps). IEEE, 2014, pp. 1433-1438.

[52] N. K. Pratas, H. Thomsen, Č. Stefanović, and P. Popovski, "Codeexpanded random access for machine-type communications," in 2012 IEEE Globecom Workshops. IEEE, 2012, pp. 1681-1686.

[53] J. H. Sørensen, E. De Carvalho, and P. Popovski, "Massive MIMO for crowd scenarios: A solution based on random access," in IEEE Globecom Workshops, 2014, pp. 352-357. 
[54] K. Au, L. Zhang, H. Nikopour, E. Yi, A. Bayesteh, U. Vilaipornsawai, J. Ma, and P. Zhu, "Uplink contention based SCMA for 5G radio access," in 2014 IEEE Globecom workshops (GC wkshps). IEEE, 2014, pp. 900-905.

[55] E. Björnson, E. De Carvalho, E. G. Larsson, and P. Popovski, "Random access protocol for massive MIMO: Strongest-user collision resolution (SUCR)," in IEEE International Conference on Communications (ICC), 2016, pp. 1-6.

[56] E. De Carvalho, E. Björnson, J. H. Sørensen, E. G. Larsson, and P. Popovski, "Random pilot and data access in massive MIMO for machine-type communications," IEEE Transactions on Wireless Communications, vol. 16, no. 12, pp. 7703-7717, 2017.

[57] H. Han, X. Guo, and Y. Li, "A high throughput pilot allocation for M2M communication in crowded massive MIMO systems," IEEE Transactions on Vehicular Technology, vol. 66, no. 10, pp. 9572-9576, 2017.

[58] J. Ding, D. Qu, H. Jiang, and T. Jiang, "Success probability of grant-free random access with massive MIMO," IEEE Internet of Things Journal, vol. 6 , no. 1, pp. 506-516, 2018.

[59] H. Jiang, D. Qu, J. Ding, and T. Jiang, "Multiple preambles for high success rate of grant-free random access with massive MIMO," IEEE Transactions on Wireless Communications, vol. 18, no. 10, pp. 4779-4789, 2019.

[60] M. B. Shahab, R. Abbas, M. Shirvanimoghaddam, and S. J. Johnson, "Grant-free non-orthogonal multiple access for IoT: A survey," IEEE Communications Surveys \& Tutorials, 2020.

[61] C. Bockelmann, H. F. Schepker, and A. Dekorsy, "Compressive sensing based multi-user detection for machine-to-machine communication," Transactions on Emerging Telecommunications Technologies, vol. 24, no. 4, pp. 389-400, 2013.

[62] F. Monsees, M. Woltering, C. Bockelmann, and A. Dekorsy, "Compressive sensing multi-user detection for multicarrier systems in sporadic machine type communication," in IEEE 81st Vehicular Technology Conference (VTC Spring), 2015, pp. 1-5. 
[63] Z. Gao, L. Dai, Z. Wang, S. Chen, and L. Hanzo, "Compressive-sensingbased multiuser detector for the large-scale SM-MIMO uplink," IEEE Transactions on Vehicular Technology, vol. 65, no. 10, pp. 8725-8730, 2015 .

[64] Y. Du, B. Dong, Z. Chen, X. Wang, Z. Liu, P. Gao, and S. Li, "Efficient multi-user detection for uplink grant-free NOMA: Prior-information aided adaptive compressive sensing perspective," IEEE Journal on Selected Areas in Communications, vol. 35, no. 12, pp. 2812-2828, 2017.

[65] H. Xiao, B. Ai, and W. Chen, "A grant-free access and data recovery method for massive machine-type communications," in IEEE International Conference on Communications (ICC), 2019, pp. 1-6.

[66] Z. Chen, F. Sohrabi, and W. Yu, "Sparse activity detection for massive connectivity," IEEE Transactions on Signal Processing, vol. 66, no. 7, pp. 1890-1904, 2018.

[67] L. Liu and W. Yu, "Massive connectivity with massive MIMO-Part I: Device activity detection and channel estimation," IEEE Trans. on Signal Processing, vol. 66, no. 11, pp. 2933-2946, 2018.

[68] L. Liu, E. G. Larsson, W. Yu, P. Popovski, Č. Stefanović, and E. De Carvalho, "Sparse signal processing for grant-free massive connectivity: A future paradigm for random access protocols in the Internet of Things," IEEE Signal Processing Magazine, vol. 35, no. 5, pp. 88-99, 2018.

[69] K. Senel and E. G. Larsson, "Device activity and embedded information bit detection using AMP in massive MIMO," in IEEE Globecom Workshops (GC Wkshps), 2017, pp. 1-6.

[70] — - "Grant-free massive MTC-enabled massive MIMO: A compressive sensing approach," IEEE Trans. on Commun., vol. 66, no. 12, pp. 6164$6175,2018$.

[71] Z. Chen, F. Sohrabi, and W. Yu, "Multi-cell sparse activity detection for massive random access: Massive MIMO versus cooperative MIMO," IEEE Trans on Wireless Commun., vol. 18, no. 8, pp. 4060-4074, 2019.

[72] D. L. Donoho, A. Maleki, and A. Montanari, "Message-passing algorithms for compressed sensing," Proceedings of the National Academy of Sciences, vol. 106, no. 45, pp. 18914-18919, 2009. 
[73] J. Kim, W. Chang, B. Jung, D. Baron, and J. C. Ye, "Belief propagation for joint sparse recovery," arXiv preprint arXiv:1102.3289, 2011.

[74] S. Haghighatshoar, P. Jung, and G. Caire, "Improved scaling law for activity detection in massive MIMO systems," in IEEE International Symposium on Information Theory (ISIT), 2018, pp. 381-385.

[75] — - "A new scaling law for activity detection in massive MIMO systems," CoRR, vol. abs/1803.02288, 2018. [Online]. Available: http://arxiv.org/abs/1803.02288

[76] A. Fengler, S. Haghighatshoar, P. Jung, and G. Caire, "Non-Bayesian activity detection, large-scale fading coefficient estimation, and unsourced random access with a massive MIMO receiver," IEEE Transactions on Information Theory, vol. 67, no. 5, pp. 2925-2951, 2021.

[77] A. Fengler, "Sparse recovery based grant-free random access for massive machine-type communication," Doctoral Thesis, Technische Universität Berlin, Berlin, 2021.

[78] A. Fengler, S. Haghighatshoar, P. Jung, and G. Caire, "Grant-free massive random access with a massive MIMO receiver," in 53rd Asilomar Conference on Signals, Systems, and Computers, 2019, pp. 23-30.

[79] S. Boyd and L. Vandenberghe, Convex Optimization. Cambridge university press, 2004.

[80] Z. Wang, Y.-F. Liu, and L. Liu, "Covariance-Based Joint Device Activity and Delay Detection in Asynchronous mMTC," arXiv preprint arXiv:2110.05815, 2021.

[81] Z. Chen, F. Sohrabi, and W. Yu, "Sparse Activity Detection in MultiCell Massive MIMO Exploiting Channel Large-Scale Fading," IEEE Transactions on Signal Processing, 2021.

[82] Z. Wang, Y.-F. Liu, Z. Chen, and W. Yu, "Accelerating Coordinate Descent via Active Set Selection for Device Activity Detection for MultiCell Massive Random Access," arXiv preprint arXiv:2104.12984, 2021.

[83] H. Iimori, T. Takahashi, K. Ishibashi, G. T. F. de Abreu, and W. Yu, "Grant-Free Access via Bilinear Inference for Cell-Free MIMO with LowCoherent Pilots," arXiv preprint arXiv:2009.12863, 2020. 
[84] T. Ding, X. Yuan, and S. C. Liew, "Sparsity learning-based multiuser detection in grant-free massive-device multiple access," IEEE Transactions on Wireless Communications, vol. 18, no. 7, pp. 3569-3582, 2019.

[85] Y. Bai, B. Ai, and W. Chen, "Deep learning based fast multiuser detection for massive machine-type communication," in IEEE 90th Vehicular Technology Conference (VTC2019-Fall), 2019, pp. 1-5.

[86] Z. Zhang, Y. Li, C. Huang, Q. Guo, C. Yuen, and Y. L. Guan, "DNNaided block sparse bayesian learning for user activity detection and channel estimation in grant-free non-orthogonal random access," IEEE Transactions on Vehicular Technology, vol. 68, no. 12, pp. 12000-12012, 2019.

[87] Y. Polyanskiy, "A perspective on massive random-access," in IEEE International Symposium on Information Theory (ISIT), 2017, pp. 25232527.

[88] A. Vem, K. R. Narayanan, J. Cheng, and J.-F. Chamberland, "A userindependent serial interference cancellation based coding scheme for the unsourced random access Gaussian channel," in 2017 IEEE Information Theory Workshop (ITW). IEEE, 2017, pp. 121-125.

[89] V. K. Amalladinne, A. Vem, D. K. Soma, K. R. Narayanan, and J.-F. Chamberland, "A coupled compressive sensing scheme for uncoordinated multiple access," arXiv preprint arXiv:1809.04745, Sept. 2018.

[90] A. Fengler, P. Jung, and G. Caire, "SPARCs for unsourced random access," IEEE Transactions on Information Theory, 2021.

[91] A. Fengler, G. Caire, P. Jung, and S. Haghighatshoar, "Massive MIMO unsourced random access," arXiv preprint arXiv:1901.00828, 2019.

[92] L. Van der Perre, E. G. Larsson, F. Tufvesson, L. De Strycker, E. Björnson, and O. Edfors, "RadioWeaves for efficient connectivity: analysis and impact of constraints in actual deployments," in 53rd Asilomar Conference on Signals, Systems, and Computers. IEEE, 2019, pp. $15-22$.

[93] P. Frenger, J. Hederen, M. Hessler, and G. Interdonato, "Antenna arrangement for distributed massive MIMO," Nov. 2019, US Patent App. $16 / 435,054$. 


\section{Included Papers}





\section{Papers}

The papers associated with this thesis have been removed for copyright reasons. For more details about these see:

https://doi.org/10.3384/9789179292218 


\section{Other Recently Published Theses From \\ The Division of Communication Systems \\ Department of Electrical Engineering (ISY) \\ Linköping University, Sweden}

Özgecan Özdogan, Signal Processing Aspects of Massive MIMO and IRS-Aided Communications, Linköping Studies in Science and Technology. Dissertations, No. 2199, 2022.

Amin Ghazanfari, Multi-Cell Massive MIMO: Power Control and Channel Estimation, Linköping Studies in Science and Technology. Dissertations, No. 2142, 2021.

Ziya Gülgün, Physcial Layer Security Issues in Massive MIMO and GNSS, Linköping Studies in Science and Technology. Licentiate Thesis, No. 1899, 2021.

Giovanni Interdonato, Cell-Free Massive MIMO: Scalability, Signal Processing and Power Control, Linköping Studies in Science and Technology. Dissertations, No. 2090, 2020.

Ema Becirovic, On Massive MIMO for Massive Machine-Type Communications, Linköping Studies in Science and Technology. Licentiate Thesis, No. 1868, 2020.

Daniel Verenzuela, Exploring Alternative Massive MIMO Designs: Superimposed Pilots and Mixed-ADCs, Linköping Studies in Science and Technology. Dissertations, No. 2041, 2020.

Trịnh Văn Chiến, Spatial Resource Allocation in Massive MIMO Communication: From Cellular to Cell-Free, Linköping Studies in Science and Technology. Dissertations, No. 2036, 2020.

Marcus Karlsson, Blind Massive MIMO Base Stations: Downlink Transmission and Jamming, Linköping Studies in Science and Technology. Dissertations, No. 1950, 2018.

Victor Hei Cheng, Optimizing Massive MIMO: Precoder Design and Power Allocation, Linköping Studies in Science and Technology. Dissertations, No. 1929, 2018.

Christopher Mollén, High-End Performance with Low-End Hardware: Analysis of Massive MIMO Base Station Transceivers, Linköping Studies in Science and Technology. Dissertations, No. 1896, 2017.

Antonios Pitarokoilis, Phase Noise and Wideband Transmission in Massive MIMO, Linköping Studies in Science and Technology. Dissertations, No. 1756, 2016. 


\section{FACULTY OF SCIENCE AND ENGINEERING}

Linköping Studies in Science and Technology,

Licentiate Thesis No. 1923, 2022

Division of Communication Systems

Department of Electrical Engineering

Linköping University

SE-581 83 Linköping, Sweden

www.liu.se 